\title{
Catalogue of Andreev spectra and Josephson effects in structures with time-reversal-invariant topological superconductor wires
}

\author{
Liliana Arrachea, ${ }^{1}$ Alberto Camjayi, ${ }^{2}$ Armando A. Aligia, ${ }^{3}$ and Leonel Gruñeiro ${ }^{1}$ \\ ${ }^{1}$ International Center for Advanced Studies, Escuela de Ciencia y Tecnología, \\ Universidad Nacional de San Martín-UNSAM, Av 25 de Mayo y Francia, 1650 Buenos Aires, Argentina \\ ${ }^{2}$ Departamento de Física, FCEyN, Universidad de Buenos Aires and IFIBA, \\ Pabellón I, Ciudad Universitaria, 1428 CABA Argentina \\ ${ }^{3}$ Centro Atómico Bariloche and Instituto Balseiro, CNEA, CONICET, 8400 S. C. de Bariloche, Argentina
}

(Dated: February 13, 2019)

\begin{abstract}
We study all the possible different two terminal configurations of Josephson junctions containing wires of time-reversal invariant topological superconductors (TRITOPS) and ordinary superconductors, including combinations with an interacting quantum dot between both wires in the junction. We introduce simple effective Hamiltonians which explain the different qualitative behaviors obtained. We analyze a wide range of phenomena, including occurrence and quenching of the so called $0-\pi$ transition, anomalous periodicity and jumps of the Josephson current as a function of the phase difference, and finite Josephson current in the absence of magnetic flux.
\end{abstract}

\section{INTRODUCTION}

Topological states of the matter is an ubiquitous topic of several areas of physics, including the communities of solid state physics,,$\frac{1}{,}$, photonic sciences, ${ }^{2]}$ and cold-atoms. ${ }^{[3}$ The branch of topological superconductivity is paramount to the field of quantum computation, after the seminal idea by Kitaev ${ }^{4}$ and coworkers 5 of exploiting the non-abelian nature of anyons. Low-dimensional topological superconductors are promising candidates for realizing topological q-bits, since they host Majorana zero-modes as edge states 67] This motivated a number of proposals to produce the topological phases in structures of quantum wires proximity-coupled to macroscopic superconductors as q-bit platforms. A prominent one is the model proposed in Refs. 8 and 9 , which is under active experimental investigation. $10-13$

The Kitaev mode ${ }^{6}$ and several other models for topological superconductivity,, 89 are based on ingredients that break time-reversal symmetry. In contrast, there is another family, the TRITOPS, which belongs to the DIII class and hosts zero-energy edge excitations that appear in Kramers pairs. So far there are no experimental realizations of this topological phase, although it is receiving significant theoretical attention in the last years. ${ }^{14}$ An interesting characteristic, of these end modes is the fact that they have fractional spin projection. ${ }^{21}$ This property can be relevant for their detection. $31 / 34 / 35 / 37]$ Recently a universal gate set using TRITOPS has been proposed. ${ }^{42}$

One of the most relevant and clear signatures of the superconducting phase is the Josephson effect, which takes place in superconductor structures with an annular shape threaded by a magnetic flux. The behavior of the generated Josephson current as a function of the phase $\phi=2 \pi \Phi / \Phi_{0}$, where $\Phi$ is the flux and $\Phi_{0}=h / 2 e$ is the superconducting flux quantum, constitutes a valuable tool to unveil interesting physics related to the nature of the superconductor or to the junction itself. In the case of ordinary superconductors, the current-phase relation has a periodicity of $2 \pi$.

In the case of junctions of topological superconducting wires, the periodicity of the Andreev spectrum is $4 \pi(2 \pi)$ if the electron parity of the system is (is not) conserved and there is a level crossing at $\phi=\pi$, which leads to a peculiar behavior of the Josephson junction. ${ }^{6 / 43}$ Josephson junctions containing one-dimensional topological superconductors with broken time-reversal (TR) symmetry have been studied in several works. Since the proposal by $\mathrm{Fu}$ and Kane for the realization of the topological phase in a quantum spin Hall system with a magnetic island, in proximity with a superconductor, the behavior of the Josephson current was suggested as a way to detect the topological phase, 44 and this system was later analyzed in other works ${ }^{45 / 46}$ Dynamical effects in Josephson junctions with topological wires with broken TR were also analyzed, ${ }^{45 / 47}$ as well as other configurations including quantum dots with many-body interactions and multiple terminals. $\$ 9$

The Josephson current in junctions of ordinary (nontopological) time-reversal invariant superconductors with an embedded interacting quantum dot (QD) has the same periodicity with the magnetic flux as the direct junctions without QD. Interestingly, they exhibit the so called $0-\pi$ transition. 68 This implies the inversion of the sign of the Josephson current, as the control parameters change and modify the occupancy and the net spin of the QD, which is a consequence of the many-body interactions and is originated in the competition of the Kondo effect with the superconducting pairing. When the former one is dominant, the system is in the 0 phase, while when the latter turns to dominate, the transition to the $\pi$-phase takes place. When the junctions are made of TRITOPS wires with an embedded interacting QD, and preserve at least one component of the spin, the peculiarity is the quenching of the $0-\pi$ transition, as discussed in Ref. 34. This is due to the screening of the magnetic moment of the quantum dot by a combination of the zero-energy modes at both sides of the quantum dot. This generates a correlated ground state and the Josephson current displays a 0-phase behavior, irrespectively of the strength of the many-body interactions in the QD. Other remarkable feature is the existence of discontinuities with large jumps in the Josephson current at both 
$\phi=0, \pi$ in junctions with one TRITOPS and another ordinary superconducting wire.28]32

The aim of the present work is the analysis and comparison of the Andreev spectrum and Josephson current of all the possible configurations containing one or two TRITOPS wires with spin-orbit interaction. We consider direct junctions and junctions with an embedded interacting QD between both wires. For the case of two TRITOPS wires, we consider the effect of different directions of the spin-orbit interaction of the two wires. We focus only on configurations which are timereversal invariant at $\phi=0, \pi$.

The work is organized as follows. Section II contains the description of the theoretical model for the wires and the effective Hamiltonian used to analyze the subgap Andreev spectrum. Results are presented in section III. Section IV is devoted to summary and conclusions.

\section{MODEL}

The full structure is modeled by the Hamiltonian $H=\sum_{\alpha} H_{\alpha}+$ $H_{J}$, where $\alpha=L, R$ labels the two wires, and $H_{J}$ is the Hamiltonian for the junction. We describe the different pieces separately

\section{A. Wires}

Each wire of the structure is modeled by the following Hamiltonian

$$
\begin{aligned}
H_{\alpha} & =\sum_{i, j}\left[\psi_{\alpha, i}^{\dagger} h_{i j}^{\alpha} \psi_{\alpha, j}+\psi_{\alpha, i}^{\dagger} \Delta_{i j}^{\alpha} \psi_{\alpha, j}^{\dagger}\right]+\text { H.c., } \\
h_{i j}^{\alpha} & =-\left(t_{\alpha}+i \lambda_{\alpha} \mathbf{n}_{\alpha} \cdot \sigma\right) \delta_{j, i+1}-\mu_{\alpha} \delta_{i, j} \\
\Delta_{i j}^{\alpha} & =\left(\tilde{\Delta}_{\alpha} \delta_{j, i+1}+\Delta_{\alpha} \delta_{i, j}\right) i \sigma_{y}
\end{aligned}
$$

where we have introduced the spinor $\psi_{\alpha, j}^{\dagger}=\left(c_{\alpha, j, \uparrow}^{\dagger}, c_{\alpha, j, \uparrow}^{\dagger}\right)$ and $\boldsymbol{\sigma}=\left(\sigma_{x}, \sigma_{y}, \sigma_{z}\right)$ are the three Pauli matrices. The terms of the normal part of the Hamiltonian are the nearest-neighbor hopping $t_{\alpha}$, and the spin-orbit interaction $\lambda_{\alpha}$ in the direction of the vector $\mathbf{n}_{\alpha}$. The pairing potential has a local s-wave component $\Delta_{\alpha}$, as well as an extended-s-wave component $\tilde{\Delta}_{\alpha}$ and $\mu_{\alpha}$ is the chemical potential.

The Hamiltonian of Eq. (1) has time-reversal symmetry and corresponds to the model introduced by Zhang-Kane-Mele in Ref. 20 for TRITOPS (DIII class) in one dimension. The key ingredients for the topological phase of class DIII are the spin-orbit interaction $\lambda_{\alpha}$ and the extended s-wave pairing $\tilde{\Delta}_{\alpha}$. The local s-wave pairing $\Delta_{\alpha}$ induces ordinary superconductivity. As discussed in Ref. 20, the TRITOPS phase takes place within the range of chemical potentials satisfying $\left|\mu_{\alpha}-\epsilon_{0, \alpha}\right|<$ $\epsilon_{m, \alpha}$, being $\epsilon_{0, \alpha}=\left(t_{\alpha} \Delta_{\alpha}\right) / \tilde{\Delta}_{\alpha}$ and $\epsilon_{m, \alpha}=2\left|\lambda_{\alpha}\right| \sqrt{1-\Delta_{\alpha}^{2} /\left(4 \tilde{\Delta}_{\alpha}^{2}\right)}$. This topological phase hosts Kramers pairs of end modes localized at the left and right edge of the wire, represented by fermionic operators $\gamma_{\alpha, s}, \tilde{\gamma}_{\alpha, s}$, with $s= \pm$, satisfying (see Ref. 35)

$$
\gamma_{\alpha,+}^{\dagger}=i \operatorname{sgn}\left(\lambda_{\alpha} \tilde{\Delta}_{\alpha}\right) \gamma_{\alpha,-}, \quad \tilde{\gamma}_{\alpha,+}^{\dagger}=-i \operatorname{sgn}\left(\lambda_{\alpha} \tilde{\Delta}_{\alpha}\right) \tilde{\gamma}_{\alpha,-} .
$$

Here,,+- denotes parallel or antiparallel orientations of the spin along the direction $\mathbf{n}_{\alpha}$.

\section{B. Junction}

We consider two types of junctions. (i) A direct junction, described by a tunneling Hamiltonian between the two wires,

$$
H_{\mathrm{J}, \mathrm{dir}}=t_{J} \sum_{s}\left(e^{i \phi / 2} c_{L, s}^{\dagger} c_{R, s}+\text { H.c. }\right) \text {. }
$$

(ii) A junction containing an interacting quantum dot embedded between the two wires

$$
\begin{aligned}
H_{\mathrm{J}, \mathrm{dot}} & =\sum_{s}\left(t_{L} e^{i \phi / 4} c_{L, s}^{\dagger} d_{s}+t_{R} e^{i \phi / 4} d_{s}^{\dagger} c_{R, s}+\text { H.c. }\right)+H_{d}, \\
H_{d} & =\varepsilon_{d}\left(n_{d, \uparrow}+n_{d, \downarrow}\right)+U n_{d \uparrow} n_{d \downarrow} .
\end{aligned}
$$

Here, $\phi=2 \pi \Phi / \Phi_{0}$, where $\Phi$ is the total magnetic flux and the index $\alpha=L, R$ corresponds to the end site of the $L, R$ wire, which intervenes in the connection of the junction.

\section{Configurations and symmetries}

The different configurations are the following: S-S, TRITOPS-TRITOPS, S-TRITOPS. The non-interacting case can be analyzed by means of exactly diagonalizing the Hamiltonian of the wires. In order to analyze the configurations with the interacting QD, we resort to low-energy effective Hamiltonians. As we will see, such effective Hamiltonians are also useful to understand most of the relevant physical properties of direct junctions.

Each wire separately has charge conjugation, time-reversal, mirror symmetry (the spin projection in the direction of the spin-orbit coupling is conserved), chiral. When they are connected having different orientations of the spin-orbit interaction, the two latter symmetries are broken. For a flux different from zero or half a superconducting flux quantum $(\phi \neq 0, \pi)$, also time-reversal symmetry is broken. For the whole system the fermion parity is a conserved quantity.

\section{LOW-ENERGY EFFECTIVE HAMILTONIANS}

\section{A. S-S}

Following Ref. 59, we define the effective low-energy Hamiltonian

$$
H_{S-S}^{\mathrm{eff}}=\sum_{\alpha}\left(\Delta_{\alpha} c_{\alpha, \uparrow}^{\dagger} c_{\alpha, \downarrow}^{\dagger}+\text { H.c. }\right)+H_{J},
$$

with $H_{\mathrm{J}} \equiv H_{\mathrm{J}, \mathrm{dir}}$, for a direct junction or $H_{\mathrm{J}} \equiv H_{J, \mathrm{dot}}$, for a junction with an embedded quantum dot. We discuss in Section IV the validity of this effective Hamiltonian. 


\section{B. TRITOPS-TRITOPS}

Following Ref. 34, we define an effective Hamiltonian where only the degrees of freedom associated to the zero modes of the topological wires are taken into account. For the case of a direct junction we have $H_{\mathrm{TRITOPS}-\mathrm{TRITOPS}}^{\text {eff }}=H_{\mathrm{J} \text {,dir }}^{\text {eff }}$, and when a dot is between the wires $H_{\mathrm{TRITOPS}-\mathrm{D}-\mathrm{efRITOPS}}^{\mathrm{eff}}=H_{\mathrm{J}, \mathrm{dot}}^{\mathrm{eff}}$. Depending on the configuration we obtain

$$
\begin{aligned}
& H_{\mathrm{J}, \mathrm{dir}}^{\mathrm{eff}}=t_{J} \sum_{s=\uparrow, \downarrow} e^{i \phi / 2} \tilde{\gamma}_{L, s}^{\dagger} \gamma_{R, s}+\text { H.c. } \\
& H_{\mathrm{J}, \mathrm{dot}}^{\mathrm{eff}}=t_{J} \sum_{s=\uparrow, \downarrow}\left(e^{i \phi / 4} \tilde{\gamma}_{L, s}^{\dagger} d_{s}+d_{s}^{\dagger} \gamma_{R, s}\right)+\text { H.c. }+H_{d} .
\end{aligned}
$$

The operators $\gamma_{\alpha, s}$ and $\tilde{\gamma}_{\alpha, s}$ are related to the Bogoliubov operators representing the zero modes defined in Eq. (2) through the transformation

$$
\begin{aligned}
& \left(\gamma_{\alpha, \uparrow}, \gamma_{\alpha, \downarrow}\right)^{T}=U_{\alpha}\left(\gamma_{\alpha,+}, \gamma_{\alpha,-}\right)^{T}, \\
& \left(\tilde{\gamma}_{\alpha, \uparrow}, \tilde{\gamma}_{\alpha, \downarrow}\right)^{T}=U_{\alpha}\left(\tilde{\gamma}_{\alpha,+}, \tilde{\gamma}_{\alpha,-}\right)^{T} .
\end{aligned}
$$

The unitary matrix relating the operators is defined from the orientation of the vector $\mathbf{n}_{\alpha}=$ ( $\sin \theta_{\alpha} \cos \varphi_{\alpha}, \sin \theta_{\alpha} \sin \varphi_{\alpha}, \cos \theta_{\alpha}$ ), as follows

$$
U_{\alpha}=\left(\begin{array}{cc}
\cos \frac{\theta_{\alpha}}{2} & -\sin \frac{\theta_{\alpha}}{2} e^{i \varphi_{\alpha}} \\
\sin \frac{\theta_{\alpha}}{2} e^{-i \varphi_{\alpha}} & \cos \frac{\theta_{\alpha}}{2} .
\end{array}\right)
$$

Without loss of generality we set $\mathbf{n}_{R}$ along the $z$-direction and $\operatorname{sgn}\left(\tilde{\Delta}_{\alpha} \lambda_{\alpha}\right)>0$. Denoting by $\tilde{\gamma}_{L,+}=\tilde{\gamma}$ and $\gamma_{R,+}=\gamma$, we have

$$
\begin{array}{llrl}
\tilde{\gamma}_{L, \uparrow}=\cos \frac{\theta}{2} \tilde{\gamma}-i e^{i \varphi} \sin \frac{\theta}{2} \tilde{\gamma}^{\dagger}, & \gamma_{R, \uparrow}=\gamma, \\
\tilde{\gamma}_{L, \downarrow}=e^{-i \varphi} \sin \frac{\theta}{2} \tilde{\gamma}+i \cos \frac{\theta}{2} \tilde{\gamma}^{\dagger}, & \gamma_{R, \downarrow}^{\dagger}=i \gamma,
\end{array}
$$

Substituting this transformation in Eqs. (6), and using the relations of Eq. (2), we get the following effective Hamiltonians

$$
\begin{aligned}
H_{\mathrm{J}, \mathrm{dir}}^{\mathrm{eff}} & =t_{0} \tilde{\gamma}^{\dagger} \gamma+\delta_{0} \tilde{\gamma} \gamma+\text { H.c., } \\
H_{\mathrm{J}, \mathrm{dot}}^{\mathrm{eff}} & =H_{L}+t_{\phi} d_{\uparrow}^{\dagger} \gamma-i t_{\phi} d_{\downarrow}^{\dagger} \gamma^{\dagger}+\text { H.c. }+H_{d} \\
H_{L} & =\sum_{s=\uparrow, \downarrow}\left(t_{s} \tilde{\gamma}^{\dagger} d_{s}+\delta_{s} \tilde{\gamma} d_{s}\right)
\end{aligned}
$$

We have defined

$$
\begin{aligned}
t_{0} & =2 t_{J} \cos \frac{\theta}{2} \cos \frac{\phi}{2}, \quad \delta_{0}=-2 t_{J} \sin \frac{\theta}{2} \sin \frac{\phi}{2} e^{i \varphi}, \\
t_{\uparrow} & =t_{\phi} \cos \frac{\theta}{2}, \quad t_{\downarrow}=t_{\phi} e^{i \varphi} \sin \frac{\theta}{2}, \quad t_{\phi}=t_{J} e^{i \phi / 4}, \\
\delta_{\uparrow} & =i t_{\phi} e^{-i \varphi} \sin \frac{\theta}{2}, \quad \delta_{\downarrow}=-i t_{\phi} \cos \frac{\theta}{2} .
\end{aligned}
$$

Notice that Eqs. 110 are actually independent of the azimuthal angle $\varphi$ as it might be expected. In fact, the latter can be eliminated by the following gauge transformation $\tilde{\gamma} \rightarrow e^{i \varphi / 2} \tilde{\gamma}, \gamma \rightarrow e^{i \varphi / 2} \gamma, d_{\uparrow} \rightarrow d_{\uparrow} e^{-i \varphi / 2}, d_{\downarrow} \rightarrow d_{\downarrow} e^{i \varphi / 2}$.

If the transformation that relates the zero modes $\gamma_{\alpha, \sigma}$ with those entering Eq. (1) is known (for example numerically in large chains or analytically as in Ref. 35$), t_{J}$ can be calculated explicitly. In the second Eq. (6) we have assumed $t_{L}=t_{R}=t$ for simplicity. In general $t_{J}$ is smaller but of the order of $t$. Since in the construction of the effective Hamiltonian the energies above the superconducting gap have been neglected, the quantitative validity of the effective Hamiltonian is restricted to $t_{J} \ll\left|\tilde{\Delta}_{\alpha}\right|,\left|\Delta_{\alpha}\right|$. The neglected terms in the derivation of the effective Hamiltonian are the hybridization of the zero modes of the opposite chain (in the case of direct junction) or of the degrees of freedom of the quantum dot (for the junction with QD), with the high-energy quasiparticles above the gap in the direct junction. Such processes can affect the parameters of the effective Hamiltonian, but do not modify its form.

\section{TRITOPS-S}

In this case, the effective Hamiltonian is a combination of the effective Hamiltonians previously formulated. Concretely, $H_{\text {TRITOPS-S }}^{\text {eff }}=H_{\mathrm{J}}+H_{S}$. The Hamiltonian $H_{J}$ of the junction corresponds to $H_{\mathrm{J}, \mathrm{dir}}^{\text {eff }}$ for a direct junction and $H_{\mathrm{J}, \mathrm{dot}}^{\mathrm{eff}}$ for a junction with a quantum dot. In the former case, it can be expressed as follows

$$
H_{\mathrm{J}, \mathrm{dir}}^{\mathrm{eff}}=t e^{i \phi / 2}\left(\tilde{\gamma}^{\dagger} c_{R,+}-i \tilde{\gamma} c_{R,-}\right)+\Delta_{R} c_{R,+}^{\dagger} c_{R,-}^{\dagger}+\text { H.c., }
$$

where we have defined $\left(c_{R,+}, c_{R,-}\right)^{T}=U_{L}\left(c_{R, \uparrow}, c_{R, \downarrow}\right)^{T}$, with $U_{L}$ given in Eq. (8). It is interesting to notice the explicit phase $\pi / 2$ in the effective pairing (2nd term of the above Hamiltonian), which is a consequence of the relations of Eq. (2) satisfied by the zero end modes. This effectively introduces a phase in the junction, in addition to the one due to the magnetic flux.

Similarly, for the case of a quantum dot embedded in the junction, we can perform the transformation $\left(d_{+}, d_{-}\right)^{T}=$ $U_{L}\left(d_{\uparrow}, d_{\downarrow}\right)^{T}$ and define $\left(c_{R,+}, c_{R,-}\right)^{T} \equiv\left(c_{R, \uparrow}, c_{R, \downarrow}\right)^{T}$. Then, the effective Hamiltonian results

$H_{\mathrm{J}, \mathrm{dot}}^{\mathrm{eff}}=H_{d}+t_{\phi}\left(\tilde{\gamma}^{\dagger} d_{+}-i \tilde{\gamma} d_{-}+\sum_{s= \pm} d_{s}^{\dagger} c_{R, s}\right)+\Delta_{R} c_{R,+}^{\dagger} c_{R,-}^{\dagger}+$ H.c.

where we are using the same definition of $t_{\phi}$ as in Eq. (12).

Notice that in this configuration, the effective Hamiltonians become independent of the orientation of the spin-orbit of the TRITOPS wire, as expected.

\section{RESULTS}

We show results calculated by the exact numerical diagonalization of the full Hamiltonian in wires of finite length $L$, along with the many-body spectrum calculated by the exact diagonalization of the effective Hamiltonians introduced in Sections III A, III B and III C. The Josephson current is calculated from

$$
J(\phi)=\frac{\partial E_{0}^{\mathrm{eff}}(\phi)}{\partial \phi}
$$


being $E_{0}^{\text {eff }}(\phi)$ the ground-state energy of the many-body spectrum calculated with the effective Hamiltonian. In the case of wires with a finite length, it can be calculated from the Matsubara Green function as follows

$$
\begin{aligned}
& J_{\mathrm{dir}}(\phi)=2 t_{J} \sum_{s} \lim _{\tau=0^{-}} \operatorname{Im}\left[\mathcal{G}_{L R, s}(\tau)\right], \\
& J_{\mathrm{dot}}(\phi)=2 t_{J} \sum_{S} \lim _{\tau=0^{-}} \operatorname{Im}\left[\mathcal{G}_{L d, s}(\tau)\right],
\end{aligned}
$$

being $\mathcal{G}_{L R, s}(\tau)=-\left\langle T_{\tau}\left[c_{L, s}(\tau) c_{R, s}^{\dagger}(0)\right]\right\rangle$ and $\mathcal{G}_{L d, s}(\tau)=$ $-\left\langle T_{\tau}\left[c_{L, s}(\tau) d_{s}^{\dagger}(0)\right]\right\rangle$. The latter can be evaluated from the results of the single-particle spectrum. We will focus on the limit of temperature $T=0$.

\section{A. Direct tunneling}

$$
\text { 1. } S-S
$$

This junction has been analyzed in several works. 59 .67 The main characteristic of the Andreev levels of this type of junction is the existence of an energy gap, which leads to a smooth behavior of the Josephson current. By comparing the results for the Josephson current obtained by the direct diagonalization of the Hamiltonian for the connected wires using Eq. (16) with those obtained from the ground-state energy of the effective Hamiltonian using Eq. (15), we verify the good qualitative agreement between the two approaches.

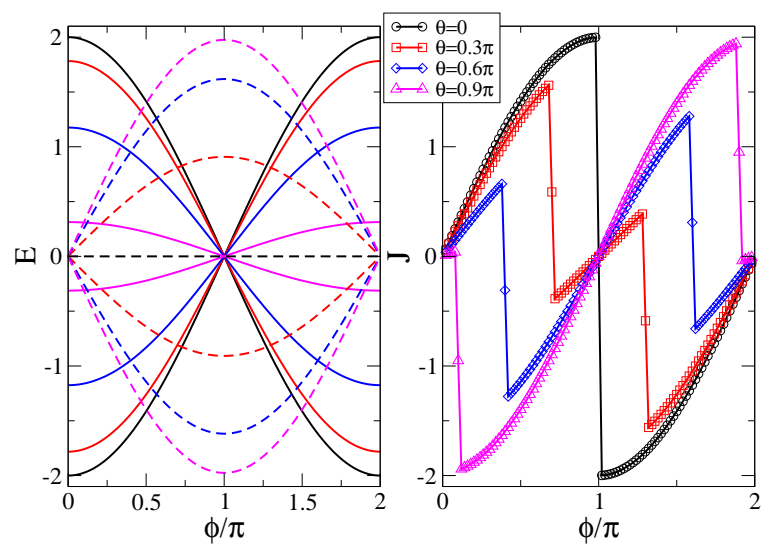

FIG. 1. Andreev spectrum of a TRITOPS-TRITOPS junction with direct tunneling between the wires calculated with the effective Hamiltonian (left) and the corresponding Josephson current calculated from Eq. (15) (right). Solid and dashed lines correspond to states within the subspace with odd and even number of particles, respectively. Different plots correspond to different relative orientation $\theta$ in the direction of the spin-orbit coupling of the wires. Energies are expressed in units of $t$.

\section{TRITOPS-TRITOPS}

The solution of the effective Hamiltonian for the junction with direct tunneling between the wires is very simple. Details of the calculation of the many-body states are presented in Appendix A The resulting spectra and Josephson current are shown in Fig. 11. We see that the effect of the relative orientation $\theta$ of the spin-orbit coupling of the two wires plays a crucial role in the behavior of the Andreev spectrum and the Josephson current. For wires with the spin-orbit coupling oriented along the same direction $(\theta=0)$, the spectrum contains only two levels with flux-dependent energies, which belong to the subspace of the effective Hamiltonian with an odd number of particles, which cross at $\phi=\pi$, while the states in the even subspace have zero energy, independently of the value of $\phi$. As a consequence of the level crossing, the Josephson current has a discontinuity at $\phi=\pi$. This feature has been already discussed in Refs. 28, 32, 34, and 36 For wires with different orientation of the spin-orbit coupling $(\theta \neq 0)$, the states within the subspace with even number of particles of $H_{\text {TRITOPS-TRITOPS }}^{\text {eff }}$ become dispersive in $\phi$. Level crossing between states with different parities take place in the intervals $0 \leq \phi \leq \pi$ and $\pi \leq \phi \leq 2 \pi$, with the consequent discontinuities in the Josephson junction.

In the limit where $\mathbf{n}_{L}$ and $\mathbf{n}_{R}$ form an angle $\theta=\pi$, the states within the subspace with odd number of particles do not disperse and have zero energy, while the ones in the even subspace fully determine the behavior of the Josephson current. An interesting feature is that, in this limit, the two dispersive Andreev states cross at $\phi=0$, leading to a discontinuity with an abrupt jump in the Josephson current at zero flux. Such a behavior is quite peculiar and has been also pointed out in other Josephson junctions of topological superconductors with broken time-reversal symmetry. ${ }^{58}$ Here, the origin is the effective phase in the junction, introduced by the different orientations of the spin-orbit interaction of the two wires. We note that when both directions coincide, the spin projection in this direction is a conserved quantity. However, this symmetry is lost in the more general case. From the exact solution of the effective Hamiltonian [Eqs. A2 and (A3], we see that the role of $\theta$ and $\phi$ can be exchanged. In particular, changing $\theta$ from 0 to $\pi$ is equivalent to a shift in $\phi$ by $\pi$. This can be understood from the relations satisfied by the zero-modes given by the generalization of Eqs. (2), when the effect of the flux is shifted to a superconducting wire by a gauge transformation [Eqs. (18), (21) and (26) of Ref. [35].

In Fig. 2, we show results for the Josephson current calculated on the basis of the exact diagonalization of wires with finite length $L$. We see that for the longer wires considered in the calculation, the results of the Josephson current reproduce all the qualitative features already shown in Fig. 1, corresponding to the effective Hamiltonian. For short lengths, the end modes within each of the TRITOPS wires hybridize and the $J(\phi)$ response departs from the description of the effective Hamiltonian, where only the zero modes directly connected to the junction are taken into account. 


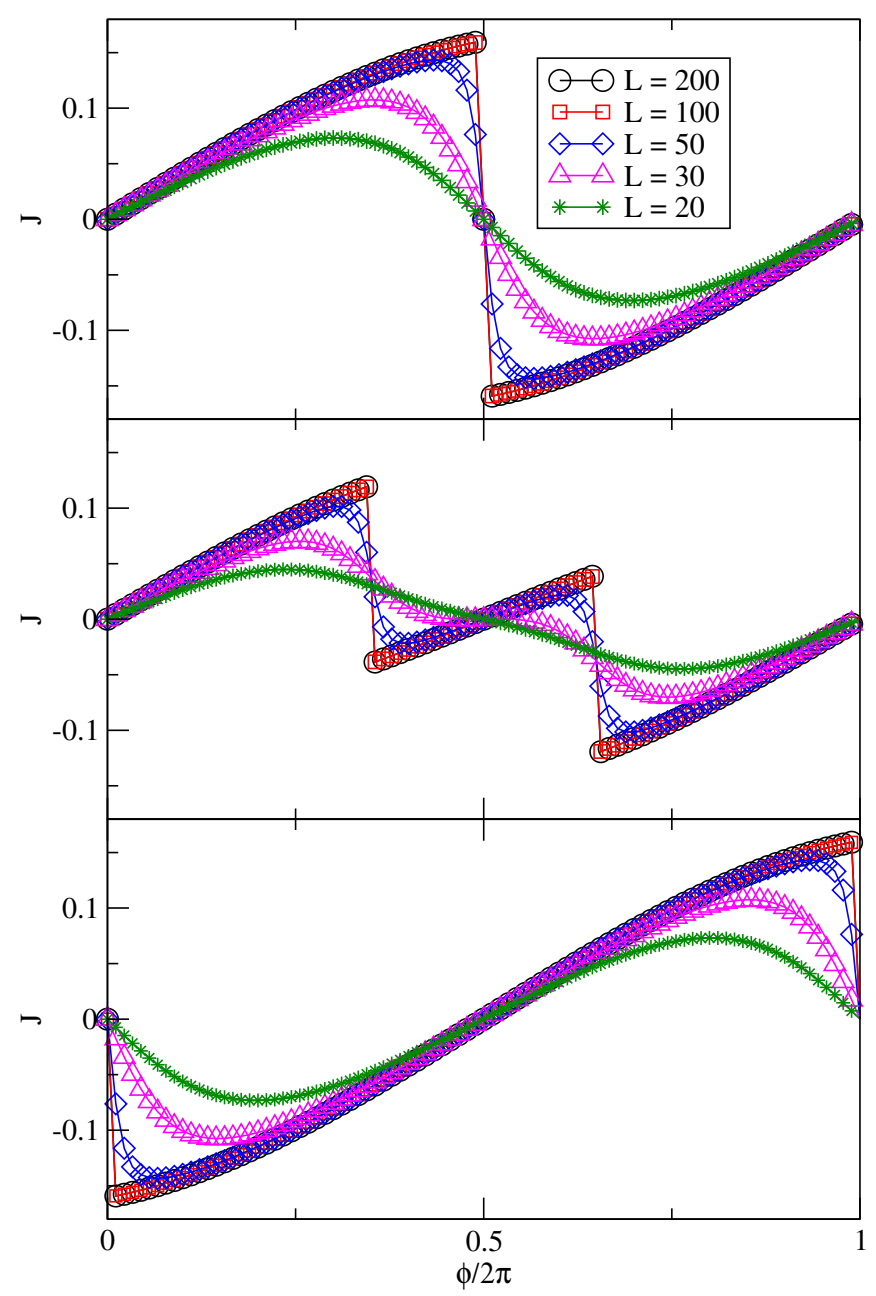

FIG. 2. Josephson current of a TRITOPS-TRITOPS junction with direct tunneling between the wires for various orientations of the spin orbit of the left wire. Results correspond to exact diagonalization of finite chains. Top, middle and bottom panels correspond to $\theta=$ $0,0.3 \pi, \pi$, respectively. Parameters are $\Delta=0, \tilde{\Delta}=0.2, \lambda=0.5, \mu=$ 0 . Energies are indicated in units of $t$.

\section{TRITOPS-S}

The solution of the effective Hamiltonian for the junction with direct tunneling between the wires is very simple. Details of the calculation of the many-body states are presented in Appendix A The resulting spectra and Josephson current are shown in Figs. 3 As mentioned in the derivation of the effective Hamiltonian, this configuration is independent of the orientation of the spin-orbit coupling. The features to highlight are: (i) the discontinuity with a jump of the Josephson current at zero flux. This is a consequence of the phase $\pi / 2$ in the effective pairing along the junction, which can be traced back to the relation between the Bogoliubov operators representing the zero modes, expressed in Eq. (2). (ii) The other

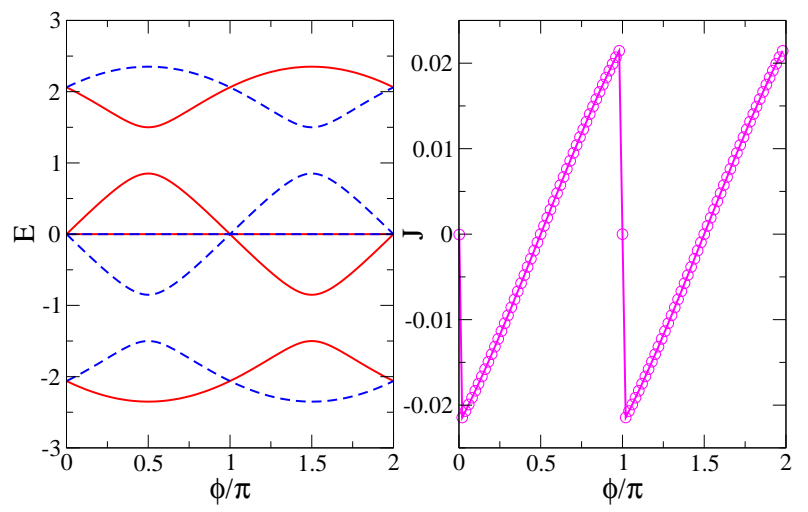

FIG. 3. Same as Fig. 1 for a TRITOPS-S junction with direct tunneling between the wires. We have considered $\Delta_{R}=1.5 t$.

interesting feature is the level crossing at $\phi=\pi$. As it is clear from the corresponding effective Hamiltonian (see appendix A), both crossings at $\phi=0$ and $\phi=\pi$ are protected by fermion parity, since the two levels that cross belong to subspaces with different parity. (iii) It is also very interesting the fact that the Josephson current seems to be approximately periodic in $\pi$ instead of in $2 \pi$. In fact, the low-energy effective Hamiltonian is exactly periodic in $\pi$ (see appendix A). In the case of wires of finite length, the coupling of zero modes modifies this picture, but the Fourier analysis of the Josephson current anyway presents a strong component associated to this half periodicity.

Results obtained by exact diagonalization of the wires are shown in Fig. 4, where the effect of the length of the wires can be appreciated.

\section{B. Junctions with embedded quantum dot}

$$
\text { 1. } S-Q D-S
$$

The description of the leads -in which only one site is considered- in the effective Hamiltonian corresponds to the atomic limit, $\sqrt[5966]{ }$ in which the superconducting gap $\left|\Delta_{\alpha}\right|$ is assumed to be much larger then the hopping term $\left|t_{\alpha}\right|$. In spite of its simplicity, as we briefly discuss below this approximation is able to describe qualitatively the $0-\pi$ transition .The occurrence of the $0-\pi$ transition is, precisely, main characteristic of this junction. It takes place as the parameters $\varepsilon_{d}$ and $U$ change with the quantum dot singly occupied. This transition has been widely discussed in the literature.5967. In Fig. 5 we review the evolution of the spectrum and the Josephson current as it takes place.

The $0-\pi$ transition is related to the Kondo effect, which consists in the formation of a singlet between the localized spin at the QD and the spins of the electrons in the wires. This effect 


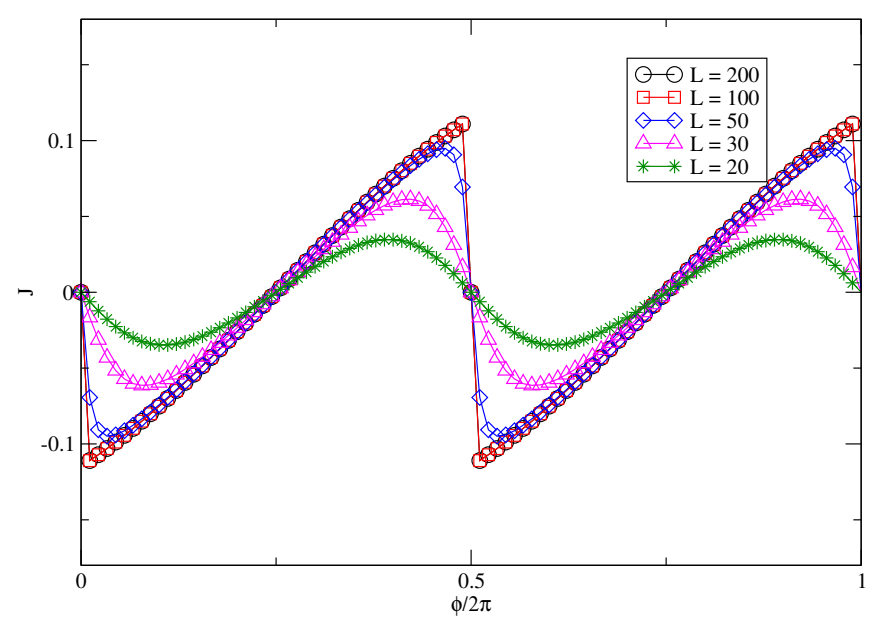

FIG. 4. Josephson current of a TRITOPS-S junction with direct tunneling between the wires. Results correspond to exact diagonalization of finite chains. In the $S$ wire $\Delta=0.5$. Parameters for the TRITOPS wire are as in Fig. 3.
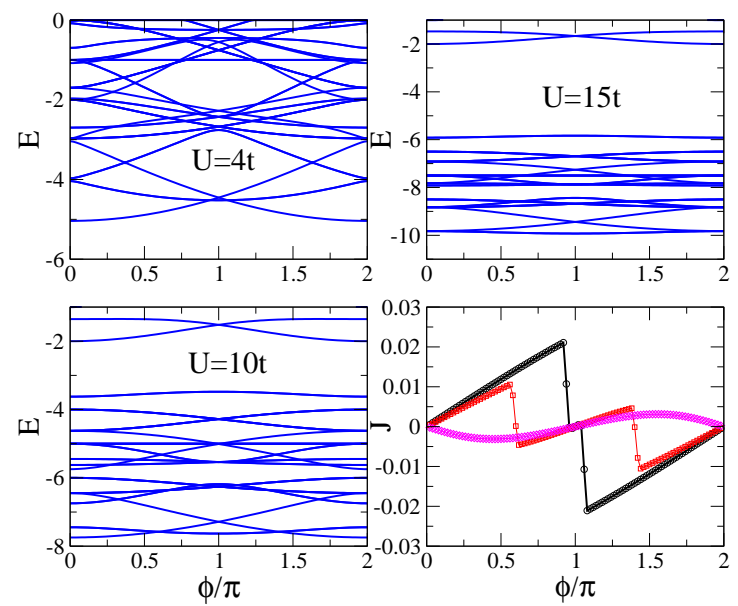

FIG. 5. Low-energy sector of the Andreev spectrum and Josephson current (bottom right) of an S-dot-S junction for different values of $U, \varepsilon_{d}=-U / 2$ and $\Delta=t$.

is characterized by the energy scale set by the Kondo temperature $T_{K}$, which in the Kondo limit $t_{\alpha} \ll-\varepsilon_{d}, t_{\alpha} \ll \varepsilon_{d}+U$ and for $\Delta \rightarrow 0$ is $k_{B} T_{K} \propto \exp [-1 /(\rho J)]$ where $\rho$ is the density of conduction states and $\left.J=2\left(t_{L}^{2}+t_{R}^{2}\right) U /\left[\varepsilon_{d}\left(\varepsilon_{d}+U\right)\right]\right)$.. Hence, in the symmetric case $\varepsilon_{d}=-U / 2$ as $U$ increases, the Kondo energy scale decreases. When $k_{B} T_{K} \ll \Delta_{R}$, it becomes energetically non-convenient to build the Kondo singlet between the localized electron and conductions electrons or holes at en- ergy $\Delta_{R}$. Then, the ground state becomes one with odd parity and an unscreened localized electron at the QD. The behavior of the ground-state energy as a function of $\phi$, changes from having a minimum at $\phi=0$ and a maximum at $\phi=\pi$ to the opposite situation, with the consequent change of sign in the Josephson current. The latter is, precisely, known as the $0-\pi$ transition.
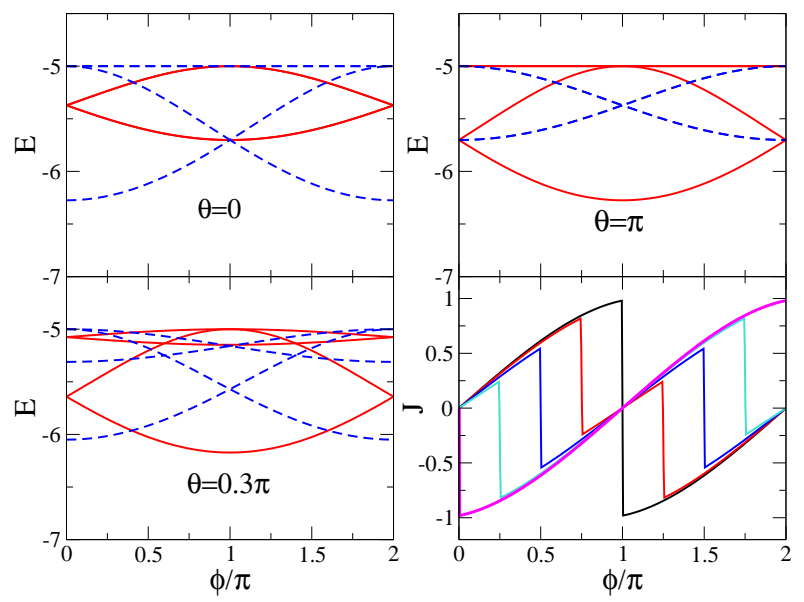

FIG. 6. Low-energy sector of the Andreev spectra of TRITOPS-QDTRITOPS junction. The Coulomb interaction and local potential of the quantum dot are, respectively, $U=10 t$ and $\varepsilon_{d}=-5 t$. Different panels correspond to different relative orientations of the spin-orbit couplings. Top right panel: the Josephson current for different values of $\theta=0, \pi / 4, \pi / 2,3 \pi / 4$ and $\pi$. Other details are the same as in Fig. 1

\section{TRITOPS-QD-TRITOPS}

Results for the Andreev spectra of a junction with an embedded quantum dot calculated by diagonalizing the effective Hamiltonian are shown in Figs. 6. Only the low-energy sector of the spectrum is shown. The case with the spin orbit of the wires oriented along the same direction $(\theta=0)$ was previously analyzed in Ref. 34 by means of a quantum Monte Carlo simulation to treat the interacting quantum dot. In addition an effective low-energy model of the Kondo type (constructed by a Schrieffer-Wolff transformation of the effective Hamiltonian) offered a simpler explanation of the main physics. The results shown in the top left panel of Fig. 6 coincide with the latter picture. The spectrum is characterized by a four-fold degenerate crossing at $\phi=\pi$. This is because, in addition to the crossing of two states belonging to the subspace with even number of particles, there is an additional crossing with a two-fold degenerate state belonging to the subspace with odd number of particles. Consequently, the Josephson current presents a discontinuity at $\phi=\pi$. This feature has been already discussed in Ref. 34 .

This behavior is strongly modified when different orientations 
of the spin-orbit coupling of the wires are considered. As in the case of the direct junction analyzed in Fig. 1, crossings between states of the different subspaces take place within the intervals $0<\phi<\pi$ and $\pi<\phi<2 \pi$. The jumps in the Josephson current take place at the crossing points. Also, as in the case of the direct junction analyzed in Section IV A 2 in the limit of $\theta=\pi$, the crossing takes place at $\phi=0$, as a consequence of the phase between the zero end-modes given in Eq. 22. This can be explicitly seen in Fig. 6 .

Overall, the behavior of the Josephson current is practically unaffected by the many-body interaction at the quantum dot. As in the case analyzed in Ref. 34 there is no signature of the $0-\pi$ transition as a function of $U$ and the occupancy of the QD. This is because the low-energy spectrum is dominated by the zero modes, which hybridize to the QD to form a combined state akin to the Kondo singlet, irrespectively of the value of $U$. In the present case, the low-energy effective Hamiltonian provides the right qualitative description of the physics (high energy perturbative processes only introduce minor corrections to the parameters).

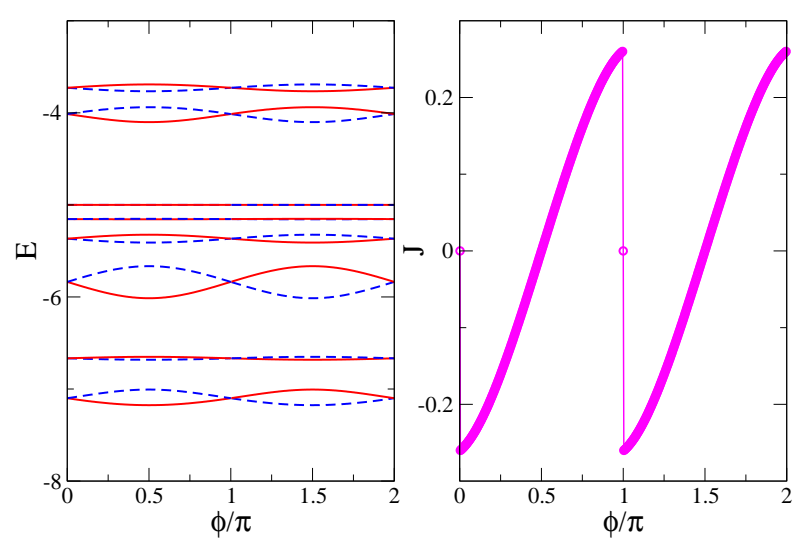

FIG. 7. Andreev spectrum (left) and Josephson current (right) of a TRITOPS-QD-S junction for $\Delta_{R}=1.5 t$. Other parameters are the same as in Fig. 6

\section{TRITOPS-QD-S}

The solution of the effective Hamiltonian of Eq. (14) for the junction between TRITOPS and $\mathrm{S}$ wires with the embedded quantum dot leads to the spectrum and Josephson current shown in Figs. 7] We see similar features as in the case of the direct coupling. In particular the features (i), (ii) and (iii) described in Section IV A 3 are also observed in the case of the junction with the embedded QD.

It is also interesting to highlight the absence of $0-\pi$ transition in this configuration. Due to the crossing of states of different fermion-parity at flux $\pi$, there is always a relative maximum in the ground state energy $E(\phi)$ there. Therefore, the $0-\pi$ transition associated with a change from a maximum of $E(\phi)$ at $\phi=\pi$ to a minimum, accompanied by a change in fermion parity from even to odd as $U$ increases, does not take place.

In the limit of $\Delta_{R} \rightarrow \infty$, we can derive the effective low-energy Hamiltonian by recourse to a Schrieffer-Wolff transformation, which reads

$$
H_{\mathrm{low}}=J\left[\left(n_{d \downarrow}-n_{d \uparrow}\right) \tilde{\gamma}^{\dagger} \tilde{\gamma}+n_{d \uparrow}\right],
$$

with $J=-\left|t_{\phi}\right|^{2} U /\left[\varepsilon_{d}\left(U+\varepsilon_{d}\right)\right]$. Therefore, as in the case of the QD connected to two TRITOPS wires, the low-energy states correspond to hybridizations of the zero modes with the QD, irrespectively of the value of $U$. The ground state does not experience any qualitative change, which explains the lack of $0-\pi$ transition as $U$ changes.

\section{SUMMARY AND CONCLUSIONS}

We have analyzed the Andreev spectrum and the Josephson current in different two-terminal configurations containing one or two TRITOPS wires with spin-orbit interaction. We have analyzed the possibility of different orientations of the spin-orbit coupling and the effect of many-body interactions when a quantum dot is embedded into the junction between both wires.

For TRITOPS-TRITOPS configurations we find that the phase introduced by the relative orientation of the spin-orbit coupling of the wires plays a role, which is similar to the one due to the magnetic flux. In this way, level crossings and the consequent jumps of the Josephson current shift from $\phi=\pi$ for parallel orientations of the spin orbit to $\phi=0$ to antiparallel ones.

For TRITOPS-S junctions, we find an abrupt discontinuity with a jump of the Josephson current at $\phi=0$ as in previous works, as well as a strong component with periodicity of half the superconducting flux quantum. ${ }^{28 \mid 32 / 36}$ These features are, however, modified in wires of finite length due to the hybridization of the zero modes.

For both cases, we find a quench of the $0-\pi$ transition in the presence of an interacting quantum dot in the junction. The reason is the hybridization of the states localized at the quantum dot with the zero modes of the TRITOPS wires, that leads to a formation of a low-energy singlet. We show that the zero mode of a single wire is enough to screen the localized state inhibiting the transition to the $\pi$ phase. In contrast, for ordinary superconductors the formation of a Kondo singlet requires taking quasiparticles with energy above the band gap. All these features can be explained in terms of simple lowenergy effective Hamiltonians.

\section{ACKNOWLEDGMENTS}

LA thanks Arbel Haim and Yuval Oreg for interesting discussions. We acknowledge support from CONICET, and UBA- 
CyT, Argentina and the Alexander von Humboldt Foundation, Germany (LA). We are sponsored by PIP 112-201501-00506 (AA), pip-rd 20141215-4905 of CONICET (LA, AC and LG) and PICT-2014-2049 (LA, AC and LG).

\section{Appendix A: Solution of $H^{\text {eff }}$ for a junction with direct tunneling}

\section{a. TRITOPS-TRITOPS}

The many-body states of $H_{\mathrm{J}, \text { dir }}^{\text {eff }}$ given in Eq. 10 can be easily constructed with the fermionic operators $\gamma$ and $\gamma$. These states are

$$
|1\rangle=|0,0\rangle,|2\rangle=|1,0\rangle,|3\rangle=|0,1\rangle,|4\rangle=|1,1\rangle,
$$

where the left and right entry denotes the occupation states of $\gamma^{\dagger} \gamma$ and $\tilde{\gamma}^{\dagger} \tilde{\gamma}$, respectively. $H_{\mathrm{J} \text {,dir }}^{\text {eff }}$ conserves the parity. In the even subspace, the two eigenenergies are

$$
E_{ \pm}^{e}(\phi)= \pm\left|\delta_{0}(\phi)\right|= \pm 2 t_{J} \cos (\theta / 2) \cos (\phi / 2)
$$

while in the odd subspace they are

$$
E_{ \pm}^{o}(\phi)= \pm t_{0}(\phi)= \pm 2 t_{J} \sin (\theta / 2) \sin (\phi / 2)
$$

\section{b. TRITOPS-S}

We proceed in a similar way as in the previous section to construct the many-body states of $H_{\mathrm{J} \text {,dir }}^{\mathrm{eff}}$ given in Eq. 13 . In the present case, the states have 3 entries, corresponding to the number states of $\gamma^{\dagger} \gamma, c_{R, \uparrow}^{\dagger} c_{R, \uparrow}$, and $c_{R, \downarrow}^{\dagger} c_{R, \downarrow}$.

$$
\begin{aligned}
& |1\rangle=|0,0,0\rangle,|2\rangle=|1,1,0\rangle,|3\rangle=|0,1,1,\rangle,|4\rangle=|1,0,1\rangle, \\
& |5\rangle=|1,0,0\rangle,|6\rangle=|0,1,0\rangle,|7\rangle=|0,0,1,\rangle,|8\rangle=|1,1,1\rangle,
\end{aligned}
$$

where the upper and lower lines correspond to the states of the even and odd subspaces, respectively. Concerning the ordering of operators $|8\rangle=\gamma^{\dagger} c_{R, \uparrow}^{\dagger} c_{R, \downarrow}^{\dagger}|0,0,0\rangle$, and the same ordering is used for the other states.

The resulting Hamiltonian matrix for the subspace with even number of particles is

$$
H_{e}=\left(\begin{array}{cccc}
0 & 0 & \Delta_{R} & i t e^{i \phi / 2} \\
0 & 0 & 0 & 0 \\
\Delta_{R} & 0 & 0 & t e^{-i \phi / 2} \\
-i t e^{-i \phi / 2} & 0 & t e^{i \phi / 2} & 0
\end{array}\right),
$$

while for odd number of particles

$$
H_{o}=\left(\begin{array}{cccc}
0 & t e^{i \phi / 2} & 0 & \Delta_{R} \\
t e^{-i \phi / 2} & 0 & 0 & -i t e^{i \phi / 2} \\
0 & 0 & 0 & 0 \\
\Delta_{R} & i t e^{-i \phi / 2} & 0 & 0
\end{array}\right),
$$

Note that the states $|2\rangle$ and $|7\rangle$ are eigenstates with energy 0 . For even number of particles, the characteristic polynomial $P_{e}(E)=\operatorname{det}\left(H_{e}-E\right)$ excluding the eigenstate $|2\rangle$ takes the form

$$
P_{e}(E)=-E^{3}+\left(\Delta_{R}^{2}+2 t^{2}\right) E-2 t^{2} \Delta_{R} \sin (\phi) .
$$

For odd number of particles, the characteristic polynomial $P_{o}(E)=\operatorname{det}\left(H_{o}-E\right)$ excluding the eigenstate $|7\rangle$ is

$$
P_{o}(E)=-E^{3}+\left(\Delta_{R}^{2}+2 t^{2}\right) E+2 t^{2} \Delta_{R} \sin (\phi) .
$$

Comparing Eqs. A6 and A7) one realizes that the spectrum for odd number of particles coincides with that for even number of particles with flux shifted by half a superconducting flux quantum $(\phi \rightarrow \phi+\pi)$. Furthermore, there is a crossing of the ground-state energies for even and odd number of particles at $\phi=0$ and $\phi=\pi$, at energy with $E=-\sqrt{\Delta_{R}^{2}+2 t^{2}}$, with the corresponding jumps in the Josephson current.
1 X-G. Wen Colloquium: Zoo of quantum-topological phases of matter Rev. Mod. Phys. 89, 041004 (2017)

${ }^{2}$ L. Lu, C. Fang, L. Fu, S. G. Johnson, J. D. Joannopoulos, and M. Soljacic Symmetry-protected topological photonic crystal in three dimensions, Nature Phys. 12, 337 (2016)

${ }^{3}$ N. Goldman, J. C. Budich, and P. Zoller, Topological quantum matter with ultracold gases in optical lattices, Nature Phys. 12, 639 (2016).

4 A. Kitaev, Fault-tolerant quantum computation by anyons, Ann. Phys. (N.Y.) 303, 2 (2003).

5 M. H. Freedman, M. Larsen, A Modular Functor Which is Universal for Quantum Computation, Z. Wang, Commun. Math. Phys. 227, 605 (2002)

6 A. Y. Kitaev, Unpaired Majorana fermions in quantum wires, Sov. Phys. Usp. 44, 131 (2001).

7 J. Alicea, New directions in the pursuit of Majorana fermions in solid state systems, Rep. Prog. Phys. 75, 076501, (2012).

8 Y. Oreg, G. Refael, and F. von Oppen, Helical Liquids and Majo- rana Bound States in Quantum Wires, Phys. Rev. Lett. 105177002 (2010)

9 R. M. Lutchyn, J. Sau, and S. Das Sarma, Majorana Fermions and a Topological Phase Transition in Semiconductor-Superconductor Heterostructures, Phys. Rev. Lett. 105077001 (2010).

10 V. Mourik, K. Zuo, S. M. Frolov, S. R. Plissard, E. P. a. M. Bakkers, and L. P. Kouwenhoven, Signatures of Majorana fermions in in hybrid superconductor-semiconductor nanowire devices, Science 336, 1003 (2012).

11 A. Das, Y. Ronen, Y. Most, Y. Oreg, M. Heiblum, and H. Shtrikman, Zero-bias peaks and splitting in an Al-InAs nanowire topological superconductor as a signature of Majorana fermions, Nat. Phys. 8, 887 (2012).

12 S. M. Albrecht, A. P. Higginbotham, M. Madsen, F. Kuemmeth, T. S. Jespersen, J. Nyg, P. Krogstrup, and C. M. Marcus, Exponential protection of zero modes in Majorana islands, Nature 531, 206 (2016).

13 M. Deng, S. Vaitiekenas, E. Hansen, J. Danon, M. Leijnse, K. 
Flensberg, J. Nygård, P. Krogstrup, and C. Marcus, Majorana bound state in a coupled quantum-dot hybrid-nanowire system, Science 354, 1557 (2016).

14 X.-L. Qi, T.L. Hughes, S. Raghu, and S.-C. Zhang, TimeReversal-Invariant Topological Superconductors and Superfluids in Two and Three Dimensions, Phys. Rev. Lett. 102, 187001 (2009).

15 L. Fu and E. Berg, Odd-parity topological superconductors: Theory and application to $\mathrm{Cu}_{x} \mathrm{Bi}_{2} \mathrm{Se}_{3}$, Phys. Rev. Lett. 105, 097001 (2010).

16 S. Ryu, A. Schnyder, A. Furusaki, and A. W. W. Ludwig, Topological insulators and superconductors: tenfold way and dimensional hierarchy, New J. Phys. 12, 065010 (2010).

17 L. Santos, T. Neupert, C. Chamon and C. Mudry, Superconductivity on the surface of topological insulators and in two-dimensional noncentrosymmetric materials, Phys. Rev. B 81, 184502 (2010).

18 C. L. M. Wong and K. T. Law, Majorana Kramers doublets in $\mathrm{d}_{x^{2} ? y^{2}}$-wave superconductors with Rashba spin-orbit coupling, Phys. Rev. B 86, 184516 (2012).

19 E. Dumitrescu, S. Tewari, Topological Properties of Time Reversal Symmetric Kitaev Chain and Applications to Organic Superconductors, Phys. Rev. B (RC) 88, 220505 (2013)

${ }^{20}$ F. Zhang, C. L. Kane and E. J. Mele, Time-Reversal-Invariant Topological Superconductivity and Majorana Kramers Pairs, Phys. Rev. Lett. 111, 056402 (2013).

21 A. Keselman, L. Fu, A. Stern, E. Berg, Inducing time reversal invariant topological superconductivity and fermion parity pumping in quantum wires, Phys. Rev. Lett. 111, 116402 (2013)

22 A. Haim, A. Keselman, E. Berg, Y. Oreg, Time-Reversal Invariant Topological Superconductivity Induced by Repulsive Interactions in Quantum Wires, Phys. Rev. B 89, 220504 (R) (2014)

${ }^{23}$ A. Haim, K. Wölms, Erez Berg, Y. Oreg, K. Flensberg, Interaction-driven topological superconductivity in one dimension, Phys. Rev. B 94, 115124 (2016)

${ }^{24}$ C. Reeg, C. Schrade, J. Klinovaja, D. Loss, DIII Topological Superconductivity with Emergent Time-Reversal Symmetry, Phys. Rev. B 96, 161407 (2017)

25 C. Schrade, A.A. Zyuzin, J. Klinovaja, and D. Loss, ProximityInduced Josephson Junctions in Topological Insulators and Kramers Pairs of Majorana Fermions, Phys. Rev. Lett. 115, 237001 (2015)

${ }^{26}$ S. Nakosai, J. K. Budich, Y. Tanaka, B. Trauzettel, and N. Nagaosa, Majorana Bound States and Nonlocal Spin Correlations in a Quantum Wire on an Unconventional Superconductor, Phys. Rev. Lett. 110, 117002 (2013).

27 S. Deng, L. Viola, and G. Ortiz, Majorana Modes in TimeReversal Invariant s-Wave Topological Superconductors, Phys. Rev. Lett. 108, 036803 (2012).

${ }^{28}$ S. B. Chung, J. Horowitz, and X-L. Qi, Time-reversal anomaly and Josephson effect in time-reversal-invariant topological superconductors, Phys. Rev. B 88, 214514 (2013).

${ }^{29}$ J. Klinovaja, A. Yacoby, and D. Loss, Kramers pairs of Majorana fermions and parafermions in fractional topological insulators, Phys. Rev. B 90, 155447 (2014).

${ }^{30}$ M. S. Scheurer and J. Schmalian, Topological superconductivity and unconventional pairing in oxide interfaces, Nature Comm. 6, 6005 (2015).

31 J. Li, W. Pan, B. A. Bernevig, R. M. Lutchyn, Detection of Majorana Kramers pairs using a quantum point contact Phys. Rev. Lett. 117, 046804 (2016).

${ }^{32}$ E. Mellars and B. Béri, Signatures of time-reversal-invariant topological superconductivity in the josephson effect, Phys. Rev. B 94, 174508 (2016).

33 W.-J. Gong, Z. Gao, W.-F. Shan, G.-Y. Yi, Influence of an embed- ded quantum dot on the josephson effect in the topological superconducting junction with Majorana doublets, Scientific reports 6 , 23033 (2016).

34 A. Camjayi, L. Arrachea, A. Aligia and F. von Oppen, Fractional spin and Josephson effect in time-reversal-invariant topological superconductors, Phys. Rev. Lett. 119, 046801 (2017).

35 A. A. Aligia and L. Arrachea, Entangled end states with fractionalized spin projection in a time-reversal-invariant topological superconducting wire, Phys. Rev. B 98, 174507 (2018).

36 A. Haim and Y.Oreg, Time-reversal-invariant topological superconductivity, arXiv: 1809.06863

37 C. Schrade and L. Fu, Parity-controlled $2 \pi$ Josephson effect mediated by Majorana Kramers pairs, arXiv:1801.03511

38 M. Mashkoori, A. G. Moghaddam, M. H. Hajibabaee, A. M. Black-Schaffer, and F. Parhizgar, Impact of topology on the impurity effects in extended s-wave superconductors with spin-orbit coupling, arXiv:1805.11885.

${ }^{39}$ F. Parhizgar and A. M. Black-Schaffer, Highly tunable timereversal- invariant topological superconductivity in topological insulator thin films, Scientific Reports 7, 9817 (2017).

40 Oscar E. Casas, Liliana Arrachea, William J. Herrera, and Alfredo Levy Yeyati, Proximity induced time-reversal topological superconductivity in $\mathrm{Bi}_{2} \mathrm{Se}_{3}$ films without phase tuning, arXiv:1812.00931

${ }^{41}$ L. Lauke, M. S. Scheurer, A. Poenicke, and J. Schmalian, Friedel oscillations and Majorana zero modes in inhomogeneous superconductors, Phys. Rev. B 98, 134502 (2018).

42 C. Schrade and L. Fu, Quantum Computing with Majorana Kramers Pairs, arXiv:1807.06620 (2017).

${ }^{43}$ H. Kwon, K. Sengupta, and V. Yakovenko, Fractional ac Josephson effect in p-and d-wave superconductors, Eur. Phys. J. B 37, 349 (2004).

${ }^{44} \mathrm{~L}$. Fu and C. L. Kane, Josephson current and noise at a superconductor/quantum-spin-Hall-insulator/superconductor junction, Phys. Rev. B 79, 161408 (2009).

45 D. M. Badiane, M. Houzet, and J. S. Meyer, Nonequilibrium Josephson effect through helical edge states, Phys. Rev. Lett. 107, 177002 (2011)

46 C. W. J. Beenakker, D. I. Pikulin, T. Hyart, H. Schomerus, and J. P. Dahlhaus, Fermion-Parity Anomaly of the Critical Supercurrent in the Quantum Spin-Hall Effect Phys. Rev. Lett. 110, 017003 (2013)

47 A. Zazunov and R. Egger, Supercurrent blockade in Josephson junctions with a Majorana wire, Phys. Rev. B 85, 104514 (2012).

48 F Pientka, A Romito, M Duckheim, Y Oreg, F von Oppen, Signatures of topological phase transitions in mesoscopic superconducting rings New Journal of Physics 15, 025001 (2013)

49 P. Marra, R. Citro, A. Braggio Signatures of topological phase transitions in Josephson current-phase discontinuities, Phys. Rev. B 93, 220507(R) (2016)

50 Y. Peng, F. Pientka, E. Berg, Y. Oreg, and F. von Oppen, Signatures of topological Josephson junctions, Phys. Rev. B 94, 085409 (2016).

51 J. I. Vaÿrynen, G. Rastelli, W. Belzig, and L. I. Glazman, Microwave signatures of Majorana states in a topological Josephson junction, Phys. Rev. B 92, 134508 (2015).

52 M. Trif, O. Dmytruk, H. Bouchiat, R. Aguado, and P. Simon, Dynamic current susceptibility as a probe of Majorana bound states in nanowire-based Josephson junctions, Phys. Rev. B 97, 041415 (2018).

53 J.Cayao, E.Prada, P.San-José, and R.Aguado, SNS junctions in nanowires with spin-orbit coupling: Role of confinement and helicity on the subgap spectrum, Phys. Rev. B 91, 024514 (2015).

54 A. Zazunov, R. Egger, and A. Levy Yeyati, Low-energy theory 
of transport in Majorana wire junctions, Phys. Rev. B 94, 014502 (2016).

55 E. B. Hansen, J. Danon, and K. Flensberg, Phase-tunable Majorana bound states in a topological N-SNS junction, Phys. Rev. B 93, 094501 (2016).

56 J. Cayao, A. M. Black-Schaffer, E. Prada, and R. Aguado, Andreev spectrum and supercurrents in nanowire-based SNS junctions containing Majorana bound states, Beilstein J. Nanotechnol. 9, 1339 (2018).

57 A. Zazunov, A. Iks, M. Alvarado, A. Levy Yeyati, and R. Egger, Josephson effect in junctions of conventional and topological superconductors, Beilstein J. Nanotechnol. 9, 1659 (2018)

58 A. Zazunov, R. Egger, T. Jonckheere, and T. Martin, Phys. Rev. Lett. 103, 147003 (2009).

59 E. Vecino, A. Martin-Rodero, and A. Levy Yeyati, Josephson current through a correlated quantum level: Andreev states and $\pi$ junction behavior, Phys. Rev. B 68, 035105 (2003).

${ }^{60}$ F. Siano and R. Egger, Josephson Current through a Nanoscale Magnetic Quantum Dot, Phys. Rev. Lett. 93, 047002 (2004).

${ }^{61}$ M.-S. Chi, M. Lee, K. Kang, and W. Belzig, Kondo effect and Josephson current through a quantum dot between two superconductors, Phys. Rev. B ( R) 70, 020502 (2004).

62 T. Meng, P. Simon, and S. Florens, Self-consistent description of Andreev bound states in Josephson quantum dot devices, Phys.
Rev. B 79, 224521 (2009).

${ }^{63}$ D. J. Luitz and F. F. Assaad, Weak-coupling continuous-time quantum Monte Carlo study of the single impurity and periodic Anderson models with s-wave superconducting baths, Phys. Rev. B 81, 024509 (2010).

${ }^{64}$ D. J. Luitz, F. F. Assaad, T. Novotny, C. Karrasch, and V. Meden, Understanding the Josephson current through a Kondo-correlated quantum dot, Phys. Rev. Lett. 108, 227001 (2012).

65 A. Oguri, Y. Tanaka, and J. Bauer, Interplay between Kondo and Andreev-Josephson effects in a quantum dot coupled to one normal and two superconducting leads, Phys. Rev. B 87, 075432 (2013).

${ }^{66}$ R. Allub and C. R. Proetto, Hybrid quantum dot-superconducting systems: Josephson current and Kondo effect in the narrow-band limit, Phys. Rev. B 91, 045442 (2015).

${ }^{67}$ G. Kirsanskas, M. Goldstein, K. Flensberg, L. I. Glazman, and J. Paaske, Yu-Shiba-Rusinov states in phase-biased superconductorquantum dot-superconductor junctions, Phys. Rev. B 92, 235422 (2015).

68 J.-D. Pillet, P. Joyez, R. Žitko, and M. F. Goffman, Tunneling spectroscopy of a single quantum dot coupled to a superconductor: From Kondo ridge to Andreev bound states, Phys. Rev. B 88, 045101 (2013). 